\title{
Corela
}

Cognition, représentation, langage

HS-1 1 | 2012

RJC Cotexte, contexte, situation

\section{Politiques linguistiques, contraintes sociolinguistiques et usages des langues dans les écoles de l'UNRWA au Liban}

\section{Myriam Abou-Samra}

\section{OpenEdition}

Journals

Édition électronique

URL : http://journals.openedition.org/corela/2280

DOI : 10.4000/corela.2280

ISSN : 1638-573X

Éditeur

Cercle linguistique du Centre et de l'Ouest - CerLICO

\section{Référence électronique}

Myriam Abou-Samra, "Politiques linguistiques, contraintes sociolinguistiques et usages des langues dans les écoles de l'UNRWA au Liban », Corela [En ligne], HS-11 | 2012, mis en ligne le 02 avril 2012, consulté le 10 décembre 2020. URL : http://journals.openedition.org/corela/2280 ; DOI : https:// doi.org/10.4000/corela.2280

Ce document a été généré automatiquement le 10 décembre 2020.

\section{(c) (i) (2)(2)}

Corela - cognition, représentation, langage est mis à disposition selon les termes de la licence Creative Commons Attribution - Pas d'Utilisation Commerciale - Partage dans les Mêmes Conditions 4.0 International. 


\title{
Politiques linguistiques, contraintes sociolinguistiques et usages des langues dans les écoles de l'UNRWA au Liban
}

\author{
Myriam Abou-Samra
}

\section{Introduction}

En nous penchant sur les pratiques langagières dans les écoles dites francophones de l'UNRWA au Liban, nous nous proposons d'analyser comment l'emploi du français comme medium d'enseignement dans ce contexte précis peut influer sur les réalisations linguistiques des apprenants et des enseignants. Avant d'analyser certaines des données qui ont été recueillies en avril 2008 dans une école de Beyrouth située dans le camp de Chatila, nous procéderons à une " mise en situation » des énoncés. Notre travail portant sur le discours de la classe, nous commencerons par définir les politiques linguistiques et éducatives à l'œuvre dans l'école dans laquelle les données ont été recueillies, en précisant les contraintes sociolinguistiques particulières qui s'exercent sur les participants du discours étudié. Considérant que la signification de l'énoncé dépend de la situation d'énonciation dont il est issu, ce n'est qu'une fois que les ingrédients du contexte auront été explicités que nous pourrons nous attarder sur leur influence sur les réalisations linguistiques. Ces dernières seront analysées en deux temps. Après avoir focalisé notre attention sur les usages du français dans le contexte scolaire, nous nous pencherons plus particulièrement sur les alternances codiques. C'est à la lumière de l'étude des interactions que nous conclurons en essayant d'apporter quelques pistes pour des propositions didactiques. 


\section{Les politiques linguistiques et le modèle d'éducation bilingue choisi : quelques précisions sur le contexte}

2 L'UNRWA est un programme des Nations-Unies créé en 1949 dont le but est de répondre aux besoins essentiels des réfugiés de Palestine en matière de santé, d'éducation, d'emploi, d'aide humanitaire et de services sociaux. Au Liban, la communauté palestinienne se caractérise par les restrictions auxquelles elle est soumise. Apatrides, les Palestiniens sont interdits de 20 professions ainsi que de l'accès à la propriété et à l'héritage ; ils n'ont pas de droits civiques et ne peuvent pas bénéficier de ce qu'offre le service public libanais, notamment en matière de santé et d'éducation. C'est donc l'UNRWA qui vient en aide aux 400.000 réfugiés palestiniens du Liban et qui scolarise 40.000 d'entre eux. Bien que l'UNRWA ait un fonctionnement qui lui est propre, elle se doit de se conformer aux lois du pays hôte. Dans le cas qui nous concerne, nous devons donc nous référer aux politiques linguistiques et éducatives libanaises.

3 Au Liban, une loi impose aux écoles publiques une répartition à part égale des heures accordées à la langue officielle (l'arabe classique) et à la langue étrangère, comprenons ici le français ou l'anglais. Concernant plus précisément les disciplines non linguistiques (désormais DNL), une loi permet le choix de la langue d'enseignement qui peut donc être l'arabe, le français ou l'anglais. Bien qu'il existe une grande liberté au niveau législatif, il faut préciser que dans les faits, seules trois écoles en 2005 ont présenté les épreuves de DNL en arabe. On peut donc dire que l'enseignement bilingue, défini comme «l'utilisation de deux (ou plusieurs) langues d'instruction à un moment donné de la carrière scolaire de l'étudiant » (Cummins, 1997) est quasi généralisé au niveau du collège.

4 L'UNRWA a choisi de calquer son programme d'éducation bilingue sur celui de l'enseignement public. Pour le présenter, nous proposons de partir la situation vécue par l'apprenant palestinien plutôt que de partir de la situation du pays, où il existe un certain nombre de cas de figure. Quand il arrive à l'école, l'apprenant palestinien parle le dialecte palestinien. Il est également plus ou moins en contact avec le dialecte libanais, le degré d'exposition variant selon les familles, le lieu d'habitation, le contexte politique, etc. La première année de l'école maternelle fonctionne en dialectes palestinien et libanais. Le français et l'arabe classique sont introduits dès la deuxième année de maternelle, cet enseignement continue à l'école primaire et au collège à raison d'environ 7 heures par semaine pour chacune des deux langues. Les DNL que sont les mathématiques et les sciences (la physique, la chimie et la biologie) sont enseignées en français à partir du CM1 et l'anglais est introduit en 5ème. À la fin de sa scolarité obligatoire, voici donc les langues qui composent le répertoire de l'apprenant:

5 Nous constatons que nous sommes ici dans une situation de diglossies imbriquées (Fasold, 1984) où les apprenants sont finalement au contact de cinq langues: le dialecte palestinien, langue de la famille, de la communauté, langue d'appartenance; le dialecte libanais, langue du pays d'accueil ; deux langues de scolarisation (le français et l'arabe) et une langue étrangère (l'anglais).

6 C'est notamment pour tenter de voir comment se manifestaient les langues en présence dans l'espace classe que nous avons procédé à des enregistrements audio de cours de français et de DNL enseignées en français. Les observations de classe se sont déroulées en 
avril 2008 sur une période de trois semaines, dans une seule école qui accueille des élèves en double vacation de $7 \mathrm{~h} 15$ à $16 \mathrm{~h} 30$, du lundi au samedi. Cela représente environ vingtcinq heures d'enregistrement, chaque enregistrement s'accompagnant d'une grille d'observation qui a été complétée pendant la séance. Des informations ont également été recueillies par le biais d'interviews qui ont été menées auprès des enseignants et des responsables éducatifs. C'est en nous référant à ces données que nous allons à présent tenter de voir comment se manifestent les langues en présence. Nous nous proposons de commencer notre analyse en nous arrêtant sur les usages du français dans la classe avant de nous pencher sur la question de l'alternance codique.

\section{Le français dans les interactions entre enseignant et apprenants : une langue de scolarisation}

7 Pour commencer, il est important d'apporter ici quelques précisions supplémentaires sur le cadre situationnel et de préciser que nous nous proposons ici d'analyser des énoncés enregistrés dans une situation d'enseignement / apprentissage. Il s'agit donc d'interactions didactiques, interactions caractérisées par le fait qu'il y ait volonté de passation d'éléments de savoir qui sont identifiables (Cicurel, 2002). En d'autres termes, un des actants - l'enseignant - possède un savoir supérieur à l'autre - l'apprenant - et désire le faire partager. Les éléments de savoir sont transmis par le biais d'un discours savant et par la mise en place d'activités pédagogiques. Dans notre cas, ce savoir concerne la langue mais également les DNL enseignées en français. En effet, bien qu'il soit quasiabsent de l'environnement social des apprenants, le français est ici objet et vecteur d'enseignement. Cette fonction de langue d'enseignement nous amène donc à considérer les réalisations linguistiques en cours de français bien sûr mais aussi en cours de DNL.

8 Notre but n'est pas ici d'analyser tous les marquages spécifiques qui font que l'interaction en classe diffère de la conversation ordinaire mais de nous arrêter sur certains éléments qui nous semblent caractéristiques de la situation étudiée. L'une des particularités du discours de classe sur laquelle nous souhaitons nous arrêter concerne la notion de bifocalisation (Bange, 1992) qui prend une nouvelle dimension dans ce contexte où il $\mathrm{y} \mathrm{a}$ acquisition simultanée du concept et de l'objet linguistique servant à l'exprimer. On se retrouve ainsi en classe de DNL, et avec certains enseignants, en présence de séquences latérales où l'attention est focalisée sur le code plutôt que sur le contenu.

L'exemple qui suit montre la manifestation d'un discours paragrammatical avec rappel du fonctionnement de la langue :

Exemple 1:

A : lampe deux et lampe trois n'a pas brillent normalement

$\mathrm{E}$ : lampe deux et lampe trois ne brillent pas normalement ne est avant le verbe pas est après

Pendant ces séquences latérales il peut également y avoir discours métalinguistique :

Exemple 2:

$\mathrm{E}:$ (...) Ţab nodros šwayet français halla 'endna deux adjectifs ici réflexion régulière

alors on va étudier un peu de français maintenant on a

et réflexion le même mot bas hon irrégulière min bi 'olni quel est l'intérêt de ce

suffixe (...)?

mais ici qui peut me dire 
11 Dans cette situation concernant la réflexion de la lumière, il y a une recherche autour de la construction des mots "régulière » et «irrégulière » entraînant une micro-activité avec la recherche d'autres couples d'antonymes :

Exemple 3 :

(...) E : min bya'tini d'autres mots?

qui peut me donner

A : complément d'objet direct complément d'objet indirect

(...)

A : article défini article indéfini

12 Ce type d'échanges assez classique en classe met en avant deux éléments qui nous semblent importants. En plus de pointer le fait que la dénomination de disciplines non linguistiques n'est pas forcément appropriée, ils rappellent que des apprenants qui utilisent le français dans un contexte scolaire uniquement ont tendance à focaliser leur attention sur l'analyse du fonctionnement de la langue. Que ce soit en cours de français ou en cours de DNL on parle de la langue, langue qui est finalement moyen et objet du discours. Les couples d'antonymes que les apprenants choisissent ici comme exemples sont tous des mots métalinguistiques, révélateurs de cette omniprésence de la réflexion linguistique dans les pratiques langagières.

13 Un autre type d'énoncés témoigne de cette centration sur le code comme on peut le voir dans les trois exemples suivants :

Exemple 4 :

$\mathrm{E}$ : comment tu sais qu'il y a un nom propre?

A : lianno fi majuscule

parce qu'il y a une

Exemple 5:

$\mathrm{E}:$ pourquoi je remplace par lui?

A : lianno singulier

parce qu'il est

Exemple 6:

$\mathrm{E}$ : pourquoi c'est un triangle isocèle? Maha leš EAB est un triangle isocèle?

pourquoi

A : lianno EB égal EA

parce que

14 Ces trois exemples présentent en fait un type d'énoncé que l'on entend très fréquemment chez les apprenants. Ces derniers tendraient à prouver que la «langue de base » (Grosjean, 1982) chez les apprenants est l'arabe mais que quelques mots sont cependant plus disponibles en français. Leur emploi répond à un besoin linguistique précis et mène à une rupture du flux discursif. "Majuscule », "singulier», "EB égal EA » relèvent finalement d'une langue de spécialité, celle du discours scolaire et sont donnés en français. Ces énoncés peuvent être expliqués par la fonction que revêt le français pour les locuteurs, une fonction qui au Liban est sujette à variation selon les locuteurs. En effet, alors que pour certains «le français est vécu non seulement comme une langue de communication mais aussi comme une langue de formation et de culture à portée identitaire », il est pour d'autres uniquement une langue de scolarisation dont l'emploi est limité à la situation de classe. L'absence de réelle communication en français peut être interprétée comme l'une des manifestations de cette étrangeté que les apprenants palestiniens ressentent vis-à-vis de la langue française.

15 En effet, parmi les représentations sur les langues les plus courantes au Liban, on trouve celle qui opère une répartition des langues en fonction des communautés religieuses. 
Ainsi les membres de la communauté chrétienne utiliseraient le dialecte libanais, le français et l'arabe littéral, le français n'étant pas ici une simple langue étrangère mais une langue que l'on pourrait qualifier de seconde, véhicule d'une certaine culture et de certaines valeurs. Les membres de la communauté musulmane utiliseraient quant à eux le dialecte libanais, l'arabe littéral et l'anglais, l'arabe étant ici la seule véritable langue de culture. La majorité des Palestiniens du Liban qui fréquentent les écoles de l'UNRWA est musulmane, on considère donc que l'anglais est pour eux une langue étrangère privilégiée. S'ajoutent à cet argumentaire le fait que la Palestine était sous mandat britannique et que l'anglais est l'une des langues de travail de l'UNRWA. En réalité, en dehors de la classe, les seules langues réellement présentes dans la communauté palestinienne sont les dialectes palestinien et libanais ainsi que l'arabe littéral. Cependant, les énoncés présentés plus haut révèlent que la classe est un espace plurilingue. Nous allons donc à présent recentrer notre attention sur les différentes langues en présence dans l'espace classe.

\section{La classe : une situation plurilingue}

Bien qu'il soit officiellement interdit d'utiliser l'arabe en classe de langue étrangère et en classe de DNL enseignées en langue étrangère, les énoncés mentionnés précédemment sont là pour attester de sa présence dans la classe. Il n'y a pratiquement pas de macroalternance, c'est-à-dire d'alternance programmée et institutionnalisée, il y a surtout de la micro-alternance (changement de langue ponctuel et non programmé au cours d'une même séquence) avec une présence dans la classe des dialectes palestinien et libanais, de l'arabe littéraire, du français et de l'anglais. Nous commencerons par l'alternance codique français-dialecte qui peut prendre des formes variées. Dans les trois exemples suivants l'enseignant parle français et l'apprenant arabe. Pourtant, l'alternance codique se déploie différemment.

Pour commencer, il arrive que l'enseignant utilise les deux langues dans un même énoncé. Très souvent, lorsque l'enseignant a recours à ce type d'alternance il emploie en français des mots qui appartiennent au registre de ce que l'on pourrait qualifier de « langue scolaire »:

Exemple 7 :

E : min ?arrab el expérience 'illi 'meln ?h ? mb ?reh bil beyt?

qui a essayé l'expérience qu'on a faite hier à la maison?

Exemple 8:

$\mathrm{E}$ : 'aš ?n h ?k sam ?n ?h ? la réflexion régulière

C'est pour ça qu'on l'a appelée

?'aš?n i?at 'ala surface plane

hein parce qu'elle est arrivée sur une

Ces exemples vont dans le même sens que ce qui a été dit précédemment et montrent que dans le discours des enseignants il y a des passages dynamiques d'une langue à l'autre au niveau intraphrasique et que l'emploi de certains mots mène à une rupture du flux discursif. Mais l'alternance français - dialecte peut se déployer différemment et il n'est pas rare que l'enseignant parle français et l'apprenant arabe, le passage d'une langue à l'autre ayant toujours lieu dans la même interaction mais le code alternant au rythme des tours de parole.

Dans le premier exemple, l'apprenant répond en arabe à une question posée en français : 
Exemple 9 :

E : Tu as lu?

A : mbareh

hier

Dans le deuxième, l'apprenant répète en arabe une question qui vient d'être posée en français :

Exemple 10 :

$\mathrm{E}$ : où est-ce qu'elle est partie?

$\mathrm{A}: \mathrm{w}$ ?n rahat?

Dans le troisième, il répète avec intonation montante ce qui vient d'être dit :

Exemple 11:

E : dix minutes

A : da'i't?n?

20 La reprise de l'énoncé de l'enseignant en arabe est pour l'apprenant un moyen de valider la compréhension de l'énoncé en français. On pourrait sans doute voir ici une stratégie d'apprentissage qui se met en place par le biais de l'alternance codique. Cependant, ces échanges peuvent aussi être considérés comme révélateurs d'une insécurité linguistique chez des apprenants qui n'osent que peu parler français et qui ont très souvent peur de ne pas comprendre. Car il est important de préciser ici que les apprenants des classes dites « francophones » sont une minorité parmi la minorité. En effet, le choix de la langue étrangère d'enseignement révèle un contraste réel entre les communautés palestiniennes et libanaises, toutes confessions confondues. Alors que 65 à $70 \%$ des élèves libanais privilégient le français, seuls $2 \%$ des élèves palestiniens ont le français comme langue étrangère 1. Ce choix s'explique en partie par l'arrière-plan que représente chacune de ces langues, arrière-plan qui révèle que le degré de xénité (Dabène, 1994) séparant les Palestiniens de la langue française est important. Le français serait alors une langue étrangère privilégiée pour les Libanais uniquement, part intégrante de l'identité libanaise mais aucunement de l'identité palestinienne.

21 Les témoignages recueillis dévoilent le fait que les enseignants eux-mêmes considèrent que les apprenants ne sont pas capables de parler français, notamment sous prétexte que c'est une langue qu'ils n'entendent pas à l'extérieur de l'école. Cette opinion transparaît lorsque l'enseignant traduit ou reformule ce qu'il vient de dire afin de, selon ses dires, pallier les difficultés de communication (supposées ou réelles) de l'apprenant:

Exemple 12:

E : comment s'appellent les amis de la poule? š ? isem rif' ?t-ha?

Comment s'appellent ses amis?

Contrairement aux exemples précédents, nous voyons qu'ici la chaîne parlée s'interrompt puisqu'il y a une boucle, une reprise de ce qui a été dit, sans doute dans le but d'assurer une compréhension immédiate et de ne pas bloquer le processus de communication.

À côté de ces alternances codiques français-dialecte, on entend également de l'anglais, même dans les classes où les apprenants n'apprennent pas encore cette langue. Nous pouvons commencer par la manière dont les apprenants interpellent les enseignantes. Au Liban, deux interpellations sont possibles, à savoir « miss » ou " demoiselle » et bien que les deux apparaissent au sein des écoles francophones de l'UNRWA, c'est la première qui est la plus récurrente - alors qu'elle est caractéristique des écoles anglophones. Vient ensuite le fait qu'il n'est pas rare d'entendre certaines lettres de l'alphabet prononcées à l'anglaise plutôt qu'à la française, [et ?] plutôt que [a ?] par exemple. Enfin, l'anglais apparaît également dans les énoncés de classe, comme on le voit dans cet extrait d'une discussion libre en classe : 


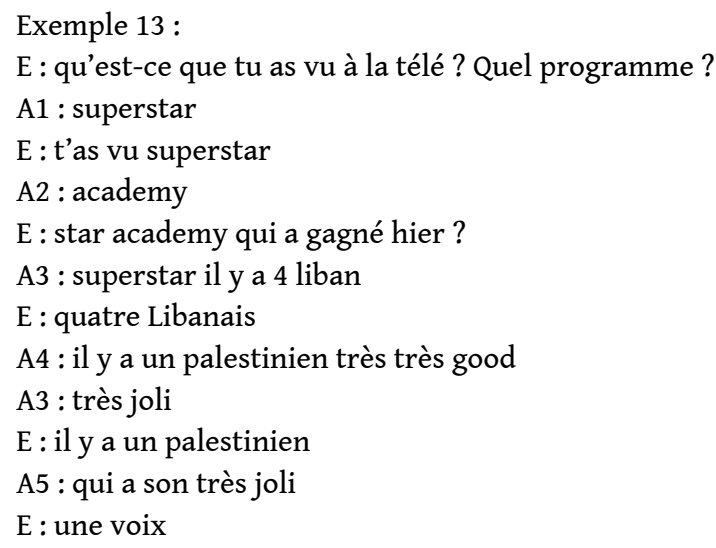

Ce phénomène peut paraître assez surprenant de prime abord, d'autant plus lorsque l'on entend de l'anglais dans les petites classes pour lesquelles l'apprentissage de cette langue n'a pas encore commencé. Il est vrai que chez les adolescents la présence de l'anglais peut s'expliquer par un effet de mode, comme un moyen d'expression de cette génération. Mais dans une certaine mesure, et sans vouloir surcontextualiser, cet emploi de l'anglais peut aussi être partie intégrante d'un processus d'affirmation de soi, les apprenants manifestant ainsi que le degré de xénité les séparant de l'anglais est moindre que celui qui les sépare du français.

Ce clivage existant entre le monde de la vie quotidienne et sociale et celui de l'école francophone se manifeste également dans les échanges où s'exprime la diglossie arabe littéral et arabe dialectal :

Exemple 14:

A : miss š ? ya3ni maquiller?

mademoiselle qu'est-ce que ça veut dire

$\mathrm{E}$ : maquiller maquillage c'est quoi maquiller? yatabara?

se maquiller

A : š ? ya'ni ? š ? ya'ni yatabara??

Qu'est-ce que ça veut dire? Qu'est-ce que ça veut dire yatabaraj?

On voit ici que l'enseignant a recours à un mot en arabe classique pour expliquer un mot en français et que ce mot pose finalement autant de difficultés de compréhension que son équivalent français. Cette réalisation linguistique nous rappelle finalement au contexte de diglossie que connaissent tous les pays arabe où coexistent une variété haute et une variété basse -voire deux- de la même langue. Ceci explique pourquoi le mot " yatabaraj ", réservé à la variété haute, n'est pas connu d'un des participants.

\section{Conclusion}

Notre objet était ici de voir comment l'emploi du français comme médium d'enseignement dans ce contexte précis pouvait influer sur les réalisations linguistiques. Nous avons pu mettre en évidence le fait que cette fonction de langue d'enseignement ne conférait pas de chef au français le statut de langue de communication. Aussi paradoxal que cela puisse paraître, ce n'est pas parce que l'enseignement de certaines disciplines se fait en français que cette langue est automatiquement parlée par les apprenants. C'est pour cette raison qu'il nous semble nécessaire de rappeler l'importance qu'il y a à articuler connaissance sur la langue et connaissance de la langue. Mais bien que peu employé par les apprenants, le français a malgré tout sa place dans la classe et le fait qu'il 
soit langue de scolarisation transparait, notamment sur le lexique. Nous rejoignons dans notre conclusion une affirmation de Lüdi et Py pour qui «c'est dans le domaine lexical justement que l'étendue et la disponibilité du vocabulaire sont liées aux circonstances personnelles du locuteur, elles-mêmes conditionnées par l'environnement culturel, social, professionnel ». Un certain nombre de phénomènes ont été relevés à travers l'analyse des alternances codiques, alternances qui sont une manifestation de la situation de contact des langues dans l'espace classe. Ces alternances codiques, qui peuvent prendre différentes formes et remplir différentes fonctions, mériteraient qu'on leur consacre plus de temps afin qu'on ne se limite pas à les interpréter comme un signe de manque de maîtrise. A terme, elles pourraient être didactisées et intégrer une démarche qui prendrait réellement en compte la compétence plurilingue des apprenants pour mieux la développer.

\section{BIBLIOGRAPHIE}

ABOU, S., HADDAD, K., KASPARIAN, C., Anatomie de la francophonie libanaise, collection Universités Francophones, série Prospectives francophones, AUPELF-UREF, Beyrouth, 1996.

BANGE, P. «A propos de la communication et de l'apprentissage en L2, notamment dans ses formes institutionnelles ", AILE, $\mathrm{n}^{\circ} 1,53-85$.

CALVET, L-J. La guerre des langues et les politiques linguistiques, Hachette Littérature, Coll. Pluriel, 2005.

CAUSA, M. L'alternance codique dans l'enseignement d'une langue étrangère, Peter Lang, 2002.

CAVALLI, M. Education bilingue et plurilinguisme : la cas du Val d'Aoste, Didier, Coll. LAL, Paris, 2005.

CICUREL, F. « La classe de langue : un lieu ordinaire, une interaction complexe, AILE $n^{\circ}$ 16, 2002.

COSTE, D. « La compétence plurilingue et ses implications possibles », in L'enseignement des langues vivantes, perspectives, Actes du séminaire organisé par la direction de l'enseignement scolaire, bureau de la formation continue des enseignants, CRDP de l'académie de Versailles, Paris, 2001, pp 29-38.

DABENE, L. Repères sociolinguistiques pour l'enseignement des langues, Hachette, Coll. FLE, Paris, 1994.

DUVERGER, J. L'enseignement en classe bilingue, Hachette, Coll. FLE, Paris, 2005.

FASOLD, R. Introduction to sociolinguistics: the sociolinguistics of society, Blackwell, 1984.

GAJO, L. \& MONDADA L. Acquisitions et interactions en contexte, Editions Universitaires, Fribourg, 2000.

GAJO, L. Immersion, bilinguisme et interaction en classe, Didier, Coll. LAL, 2001.

HAFEZ, A. Statuts, emplois, fonction, rôles et représentations du français au Liban, L'Harmattan, Paris, 2006.

LUDI, G. « Parlers bilingues et traitements cognitifs », Intellectica, 1995/1, 20, pp 139-156. 
MONDADA, L. \& PEKAREK DOEHLER, «Interaction sociale et cognition située : quels modèles pour la recherche sur l'acquisition des langues », AILE, $\mathrm{n}^{\circ} 12,2000$.

PY, B. « Bilinguisme, exolinguisme et acquisition : rôle de L1 dans l'acquisition de L2 », TRANEL n ? 17, 1991.

PY, B. « Apprendre une langue et devenir bilingue : un éclairage acquisisionniste sur les contacts de langues ", Journal of Language contact, thema 1, 2007.

SAYIGH, R. Too many enemies: the Palestinian experience in Lebanon, Zed Books, 1994.

VERDELHAN, M., Le français de scolarisation : pour une didactique réaliste, PUF, 2002

\section{RÉSUMÉS}

En nous intéressant à l'usage des langues dans les écoles de l'UNRWA au Liban, nous développerons la notion de contexte selon une approche spatiale et socioculturelle. Nous nous proposons ici d'analyser, à la lumière de données sociopolitiques et sociolinguistiques, comment l'emploi du français comme médium d'enseignement dans ce contexte précis de la scolarisation des réfugiés palestiniens peut influer sur les réalisations linguistiques. Pour ce faire, nous nous appuierons sur des observations de classes, des textes officiels, des manuels et des témoignages d'apprenants et d'enseignants.

Studying the use of languages in UNRWA's schools in Lebanon, we are going to develop the notion of context according to a spatial and sociocultural approach. In the light of sociopolitical and sociolinguistic data, we are going to analyze how the teaching of curricular subjects through the medium of French in this context of the schooling of the Palestinian refugees can influence the linguistic realizations. In order to do so, we are going to base our work on class observations, official texts, textbooks and accounts given by pupils and teachers.

\section{INDEX}

Mots-clés : politiques linguistiques, enseignement bilingue, interactions de classe, alternance codique, Liban

Keywords : language policies, bilingual education, classroom interactions, code switching, Lebanon

\section{AUTEUR}

\section{MYRIAM ABOU-SAMRA}

Université Paris 3 - Sorbonne Nouvelle 